\title{
Spinal Cord-Midbrain Functional Connectivity Is Related to Perceived Pain Intensity: A Combined Spino-Cortical fMRI Study
}

\author{
Christian Sprenger, Jürgen Finsterbusch, and Christian Büchel \\ Department of Systems Neuroscience, University Medical Center Hamburg-Eppendorf, 20246 Hamburg, Germany
}

The dynamic interaction between ascending spinocortical nociceptive signaling and the descending control of the dorsal horn (DH) by brain regions such as the periaqueductal gray matter $(\mathrm{PAG})$ plays a critical role in acute and chronic pain. To noninvasively investigate the processing of nociceptive stimuli in humans, previous fMRI studies either focused exclusively on the brain or, more recently, on the spinal cord. However, to relate neuronal responses in the brain to responses in the spinal cord and to assess the functional interplay between both sites in normal and aberrant conditions, fMRI of both regions within one experiment is necessary. Employing a new MRI acquisition protocol with two separate slice stacks, individually adapted resolutions and parameter settings that are dynamically updated to the optimized settings for the respective region we assessed neuronal activity in the spinal cord and in the brain within one measurement at $3 \mathrm{~T}$. Using a parametric pain paradigm with thermal stimulation to the left radial forearm, we observed BOLD responses in the ipsilateral $\mathrm{DH}$ of the spinal segment $\mathrm{C} 6$ and corresponding neuronal responses in typical pain-processing brain regions. Based on correlations of adjusted time series, we are able to reveal functional connectivity between the spinal C6-DH and the thalamus, primary somatosensory cortex, bilateral insula, bilateral striatum, and key structures of the descending pain-modulatory system such as the PAG, the hypothalamus, and the amygdala. Importantly, the individual strength of the spinal-PAG coupling predicted individual pain ratings highlighting the functional relevance of this system during physiological pain signaling.

Key words: fMRI; functional connectivity; PAG; pain; spinal cord

\section{Introduction}

Extensive evidence indicates the dorsal horn (DH) of the spinal cord is not only the first synapse within the afferent pain pathway but is also a crucial target for descending modulatory networks originating from brain regions such as the periaqueductal gray matter (PAG). The mutual interplay between bottom-up and top-down signaling therefore represents a fundamental dimension in pain processing (Fields, 2004; Heinricher et al., 2009).

To investigate the processing of nociceptive stimuli in humans, previous studies frequently used fMRI of the brain while fMRI of the human spinal cord became reliable only in recent years, as it is technically more challenging (Giove et al., 2004; Summers et al., 2010; Stroman et al., 2014; Wheeler-Kingshott et al., 2014). Nevertheless, spinal fMRI provides the unique opportunity to record neuronal responses at the site of signal entry into the CNS and has enabled important insights into human neurophysiology (Mainero et al., 2007; Eippert et al., 2009; Sprenger et

Received Dec. 2, 2014; revised Feb. 3, 2015; accepted Feb. 4, 2015.

Author contributions: C.S. and C.B. designed research; C.S. and J.F. performed research; J.F. contributed unpublished reagents/analytic tools; C.S. and C.B. analyzed data; C.S., J.F., and C.B. wrote the paper.

C.S. and C.B. are supported by the European Research Council grant ERC-2010-AdG_20100407.

The authors declare no competing financial interests.

Correspondence should be addressed to Christian Sprenger, Department of Systems Neuroscience, University Medical Center Hamburg-Eppendorf, Martinistrasse 52, 20246 Hamburg, Germany. E-mail: c.sprenger@uke.de.

DOI:10.1523/JNEUROSCI.4897-14.2015

Copyright $\odot 2015$ the authors $\quad 0270-6474 / 15 / 354248-10 \$ 15.00 / 0$ al., 2012; Geuter and Büchel, 2013; Nash et al., 2013; Barry et al., 2014). However, given strong evidence indicating the DH as a key site for nociceptive modulation by descending networks (Reynolds, 1969; Villanueva and Le Bars, 1995; Millan, 2002; Porreca et al., 2002; Fields, 2004; Eippert et al., 2009; Sprenger et al., 2012; Büchel et al., 2014), studies directly relating neuronal responses in the spinal cord to responses in the brain and assessing the functional connectivity (Friston et al., 1997, 2003) between both would be very informative with regard to key issues of pain processing. For example, this would allow studying the $\mathrm{DH}$ as a part of a recurrent hierarchical system and to characterize top-down or bottom-up modulation of neuronal activity more comprehensively in health and pathological conditions, which has been recently proposed in the context of a predictive coding framework (Büchel et al., 2014).

This, however, requires sampling responses in the brain and in the spinal cord within a single measurement. Such combined acquisitions are challenging due to the anatomical distance that hampers an optimal shim adjustment for both regions and considerable differences in the suitable parameter settings for both regions. To this end we recently developed a new acquisition protocol (Finsterbusch et al., 2013) that allows the consecutive acquisition of brain and cervical spinal cord activity within an appropriate repetition time. It comprises two slice stacks that can be individually positioned, slice thicknesses, and in-plane resolutions that are adapted to the desired settings for each region and 
hence an optimized sequence timing. To ensure the best possible shim adjustment for both regions a dynamic update of resonance frequency and linear shim terms is realized (Blamire et al., 1996).

Employing a parametric heat pain paradigm with two distinct temperatures in healthy participants we were able to test for painrelated BOLD responses in the brain and in the spinal cord and to compare them with findings from previous studies. Using seedbased correlations of adjusted time series allowed us to assess the functional connectivity between the involved $\mathrm{DH}$ in the spinal cord and pain-processing regions in the brain.

\section{Materials and Methods}

Participants. Twenty healthy participants ( 12 male, 8 female; mean age: 26.6 years; range: $22-35$ years) took part in this study. Exclusion criteria were abnormal heat pain threshold at the site of stimulus application, any history of neurological disease including any history of pain syndrome, current medication (excluding contraceptives), and any pain event within the last week. Data from 3 of the 20 participants had to be discarded due to imaging artifacts (1 participant) and technical malfunction (2 participants). The study was conducted in accordance with the Declaration of Helsinki and approved by the Ethics Committee of the Medical Council of Hamburg.

Experimental paradigm. After participants were informed about the study and gave written informed consent, we started with an assessment of the heat pain threshold according to the quantitative sensory testing protocol of the German Research Network on Neuropathic Pain (Rolke et al., 2006).

The experimental paradigm consisted of painful thermal stimulation with two different intensities ( 46.0 and $47.0^{\circ} \mathrm{C}$ ) applied to the left radial forearm, an area that is innervated by the nervus cutaneous antebrachii lateralis (dermatome C6). Heat stimulation was performed using a $30 \times$ $30 \mathrm{~mm}^{2}$ Peltier thermode (TSA-II; Medoc). We used Presentation software (Neurobehavioral Systems) for stimulus control and recording of pain ratings. Each of the three sessions $(\sim 18.5 \mathrm{~min})$ consisted of eight high-intensity and eight low-intensity trials applied in a pseudorandomized order. Each single trial consisted of an anticipation period, painful heat stimulation, a rating period, and finally a variable intertrial interval (ITI). At the start of the anticipation phase, a white crosshair changed its color to red, which signaled that painful stimulation would follow soon. After a variable duration ( $5 \pm 1 \mathrm{~s})$, a $15 \mathrm{~s}$ thermal stimulus was administered ( $\sim 2.5 \mathrm{~s}$ ramp up, $10 \mathrm{~s}$ plateau, and $\sim 2.5 \mathrm{~s}$ ramp down). After a delay of $10 \mathrm{~s}$ subjects had to rate the level of pain present during that trial using a visual analog scale (VAS; endpoints labeled with no "pain" and "unbearable pain"). Finally, a variable ITI $(35 \pm 5 \mathrm{~s})$ followed, during which a white crosshair was displayed.

MRI data acquisition. Imaging data were acquired on a Siemens TIM Trio 3 tesla system equipped with a 12-channel head coil combined with a 4-channel neck coil (both receive only). Participants were positioned with the isocenter of the magnet aligned with vertebral-level C2 in axial alignment avoiding strong extension or flexion of the neck. Foam pads were inserted to minimize movements of the head and neck.

A full description of the MRI pulse sequence and acquisition strategy can be found in Finsterbusch et al. (2013). Forty slices in total divided into two subvolumes were acquired in descending order. The upper subvolume covered 32 slices in the brain. Slice orientation was tilted individually by $\sim 15^{\circ}$, which allowed us to measure BOLD responses in most parts of the brain including the midbrain and the upper part of the cerebellum. The lower subvolume consisted of eight slices oriented approximately perpendicular to the spinal cord covering the lower part of the fourth, the fifth, and the upper part of the sixth cervical vertebral body, therefore, centered at the spinal segment C6 (Kim et al., 2012).

Importantly, MRI acquisition parameters were individually adapted to the favored setting for each region. Slices in the brain had an FOV of $224 \times 256 \mathrm{~mm}^{2}$, a voxel size of $2.0 \times 2.0 \times 2.0 \mathrm{~mm}^{3}$, and a gap between slices of $1.0 \mathrm{~mm}$. For the slices in the spinal cord, the FOV was set to $112 \times 128 \mathrm{~mm}^{2}$ with a voxel size $1.0 \times 1.0 \times 5.0 \mathrm{~mm}^{3}$ (without gap between slices) to achieve an adequate SNR despite realizing a high inplane resolution.
Two saturation pulses were applied anterior and posterior to the target region in a $\mathrm{V}$-shaped configuration to minimize ghosting and inflow artifacts related to pulsatile blood flow in the major cervical vessels.

A gradient echo EPI sequence with different timings for the two subvolumes was used to provide optimized parameters for the different resolutions. The bandwidths per pixel were 1502 and $1086 \mathrm{~Hz}$, yielding echo spacings of 0.75 and $1.05 \mathrm{~ms}$ for the brain and spinal cord slices, respectively.

Flow-rephasing gradient pulses were applied in the slice direction to minimize signal variations related to pulsatile flow of the CSF (Schroth and Klose, 1992a, b; Giove et al., 2004).

To minimize signal intensity variations along the $z$-direction (Cooke et al., 2004; Maieron et al., 2007) a compensatory slice-specific gradient momentum was used for the spinal slices (Finsterbusch et al., 2012). This " $z$-shim" was determined based on prescan acquisitions of the spinal subvolume with 21 equidistant gradient steps applied to all spinal slices and, subsequently, selecting the gradient setting yielding the maximum signal intensity within the spinal cord in each slice.

Parallel imaging using GRAPPA with an acceleration factor of 2 and 48 reference lines was used to minimize echo times and geometric distortions. Thus, echo train lengths of 41 and $58 \mathrm{~ms}$ and echo times of 30 and $38 \mathrm{~ms}$ could be achieved for brain and spinal cord slices, respectively. Fat saturation pulses were applied with a frequency offset of $407 \mathrm{~Hz}$ yielding acquisition times of 78 and $95 \mathrm{~ms}$ per slice for the brain and the spinal cord slices, respectively. Consequently, a repetition time of $3270 \mathrm{~ms}$ was obtained for the measurement of all 40 slices.

As the optimal shim adjustment differs substantially between the brain and the spinal cord, a dynamic update of the resonance frequency and the linear shims (Blamire et al., 1996; Morrell and Spielman, 1997) was performed during the EPI measurements. Resonance frequency and linear shim terms were dynamically adapted to the optimized values for the brain and the spinal cord subvolume, respectively. The second-order shims could not be dynamically changed on our MR system, and, thus, remained constant during the acquisitions and were determined for a large volume comprising both subvolumes.

With this approach we obtained an overall good image quality (Finsterbusch et al., 2013). However, in some cases image artifacts related to a nonoptimal resonance frequency appeared in the brain, which could be minimized by a readjustment of the frequency offset for the brain slices (six participants). In two of these cases the required offsets yielded a frequency for the fat saturation applied for the brain slices that was close to the water resonance frequency in the spinal cord leading to a significant signal loss in the spinal slices. In these cases we applied the fat saturation pulses for the brain slices without the frequency offset, i.e., with the same frequency as the fat saturation in the spinal cord.

As the head coil elements contribute considerable noise but hardly any signal to the spinal slices and this applies to the neck coil elements and vice versa, only the signal of the head coil elements was considered for the slices in the brain, and only the data of the neck coil elements were used for the spinal slices.

Two image reconstructions were performed successively, one with the reconstruction parameters for the spinal cord subvolume, the other with the parameters for the brain, to obtain optimal reconstruction results for both regions (Finsterbusch et al., 2013). Subsequently, DICOM files were converted into the NIfTI format and the brain and the spinal subvolumes were stored as two separate files. For all analyses regarding the brain we used the brain-optimized reconstructed images and for all analyses regarding the spinal cord we used the images reconstructed with the parameters for the spinal subvolume.

High-resolution $\left(1 \times 1 \times 1 \mathrm{~mm}^{3}\right)$ T1-weighted anatomical images were acquired using a 3D-MPRAGE sequence (sagittal slice orientation, repetition time $2.3 \mathrm{~s}$, echo time $3.45 \mathrm{~ms}$, flip angle $9^{\circ}$, inversion time $1.1 \mathrm{~s}$, and FOV $192 \times 240 \times 320 \mathrm{~mm}^{3}$ ). The FOV covered the entire head and neck up to the upper part of the third thoracic vertebra.

Finally, we also acquired $\mathrm{T} 2{ }^{\star}$-weighted anatomical images of the spinal target region using a MEDIC sequence (eight transversal slices, slice thickness $5 \mathrm{~mm}, 1 \times 1 \mathrm{~mm}^{2}$ in-plane resolution, repetition time $315 \mathrm{~ms}$, echo time $22 \mathrm{~ms}$, flip angle $20^{\circ}$, FOV $256 \times 184 \times 40 \mathrm{~mm}^{3}$, GRAPPA 
acceleration factor 2 , and 24 reference lines). Slices were positioned identical to the spinal slices of the functional acquisitions (see above).

Physiological data acquisition. To allow for retrospective physiological noise correction, which is crucial in spinal fMRI (Brooks et al., 2008; Piché et al., 2009; Kong et al., 2012), the pulse oxymetric pulse curve (from the left index finger), electrocardiography, and respiratory chest movements (using a respiratory belt) were measured using a clinical monitoring unit (Expression; In Vivo). Data were recorded together with the scanner pulses using Spike2 software (Cambridge Electronic Design) to ensure timing accuracy.

Behavioral data analysis. All behavioral data were analyzed in MATLAB 7.7 (The MathWorks). We calculated the mean pain ratings across the entire experiment for both intensities separately. To test for a difference in the perceived pain intensity between the high- and low-intensity conditions, we used a paired $t$ test (one-tailed). Results were considered significant at $p<0.05$.

$f M R I$ data preprocessing. $\mathrm{fMRI}$ data preprocessing and statistical analyses were performed using SPM8 (Wellcome Trust Centre for Neuroimaging, London, UK). The preprocessing of brain and spinal EPIs was performed separately following established procedures for the respective region. Generally, the first five EPIs were discarded to eliminate T1 saturation effects.

The preprocessing of brain EPIs comprised slice time correction, realignment (rigid body motion correction with 6 degrees of freedom), spatial normalization to the MNI space using the SPM EPI template, and finally smoothing with an $8 \mathrm{~mm}$ FWHM isotropic Gaussian kernel. For combined acquisitions the SPM8 standard normalization approach to the EPI template yields better results compared with the application of parameters obtained from an iterative segmentation-normalization process of the corresponding T1-weighted anatomical data (Ashburner and Friston, 2005; Finsterbusch et al., 2013).

The preprocessing of the spinal cord EPIs started with a motion correction procedure using a rigid body transformation with 6 degrees of freedom. While respiration-related $\mathrm{B}_{0}$ variations due to the proximity to the lungs might cause artificial nonrigid movements of the spinal cord when acquiring large craniocaudal portions of the spinal cord, the assumption of rigid body movements of the spinal cord appears to be justified when acquiring only a limited craniocaudal extension (Verma and Cohen-Adad, 2014). To minimize influences such as movements of neck muscles or swallowing on the motion-correction procedure, the cost function was weighted by an automatically created binary mask (using MATLAB's Image Processing Toolbox) that only included the spinal cord and directly surrounding tissues. Within this mask, highly variant regions (i.e., CSF) were excluded, to minimize the influence of strong-intensity fluctuations on the motion-correction procedure and to base the image registration only on spinal cord movements.

Next, we coregistered the anatomical T2*-weighted MEDIC images to the mean spinal EPIs ( 6 degrees of freedom) using normalized crosscorrelation as the objective function. Subsequently, we coregistered the anatomical T1-weigthed MPRAGE images to the MEDIC images using normalized mutual information as objective function (Collignon et al., 1995). This two-step coregistration procedure yields better results compared with a direct coregistration of the T1-weigthed anatomical images to the mean spinal EPI. However, if the coregistration of anatomical and functional images was suboptimal we manually optimized this step (five cases).

We next spatially normalized the data. To this end we created an anatomical template of the entire head and neck. We therefore coregistered the mean group structural image of a previous spinal cord fMRI study (Sprenger et al., 2012) to the mean group structural image of a previous whole-brain fMRI study (Sprenger et al., 2011), adjusted the intensity of both images, and merged them to one image. Subsequently, we normalized all individual T1-weigthed images to this template using the SPM normalization algorithm (including both affine and nonlinear terms, where nonlinear terms are modeled by a linear combination of discrete cosine basis functions). The normalized T1-weighted images were then averaged to build a mean T1-weighted group image. Subsequently, we also spatially normalized the spinal EPIs and the $\mathrm{T} 2{ }^{\star}$ weighted MEDIC images by applying the parameters obtained from the normalization of the T1-weigthed images and resampled images with a resolution of $1 \times 1 \times 1 \mathrm{~mm}^{3}$.

Finally, the spinal functional images were smoothed with a $2 \mathrm{~mm}$ (FWHM) isotropic Gaussian kernel to facilitate group analysis, but at the same time to allow an appropriate anatomical assignment within the spinal cord.

Statistical fMRI data analysis. The statistical fMRI data analysis was performed using a general linear model approach separately for the brain and the spinal cord subvolume. The first-level design matrix was kept, however, identical for both subvolumes and included regressors for the experimental conditions "anticipation," "low pain intensity," "high pain intensity," "VAS rating," and a session constant. As there is a considerable influence of physiological noise at the spinal level (Giove et al., 2004; Stroman, 2005; Brooks et al., 2008; Piché et al., 2009; Kong et al., 2012), the first-level design matrix additionally included regressors to correct for this potential confound. As in previous studies (Eippert et al., 2009; Sprenger et al., 2012), we followed the approach described previously (Deckers et al., 2006). This and all subsequent first-level analyses were also subjected to temporal high-pass filtering (cutoff period: $128 \mathrm{~s}$ ). Regressors representing the experimental paradigm were then modeled by convolving boxcar functions for each regressor with the canonical HRF. After model estimation we defined adequate first-level contrasts to test for (1) low pain intensity, (2) high pain intensity, (3) high minus low intensity, and (4) low minus high intensity. The ensuing contrast images from each subject were used for second-level analyses using one-sample $t$ tests to test for $1-4$.

To assess the functional connectivity between the spinal cord and the brain we implemented a seed-based connectivity analysis by correlating the time course of the spinal DH with the time courses of all brain voxels. This was performed by a GLM analysis that also accounted for taskinduced effects. We therefore extracted the time series from the spinal peak voxel of the "main effect of pain" contrast and the "high minus low" contrast. These spinal cord time series (adjusted for effects of interest and mean centered) were then used as regressors in a new first-level model for the brain data. To account for systematic stimulus/task-induced variance and to avoid the confound of activation-induced correlations (Poldrack et al., 2011), we also included all box-car regressors from the initial GLM analysis. As the estimation of functional connectivity can be influenced by physiological noise (Birn, 2012), the regressors representing the physiological noise modeling (see above) were also included to minimize this potential confound. Thus the final design matrix was identical to the initial GLM analysis except that it additionally contained the times series from the respective seed voxel from the spinal cord. In the remainder of this paper, we will refer to this as "time series correlation." After model estimation we raised the parameter estimates from this analysis to a second-level group analysis using a one-sample $t$ test.

Results were considered significant at $p<0.05$, corrected for multiple comparisons using the FWE rate. Approaches adjusting $p$ values to control FWE rates based on the Gaussian random field theory are not suitable for extremely small cylindrical structures such as the spinal cord, as the smoothness estimation that forms the basis of Gaussian random field correction for multiple comparisons is very conservative and thus leads to increased false negatives (Worsley et al., 1992; Brooks, 2014). We therefore used nonparametric permutation tests (Nichols and Holmes, 2002) using the Statistical Non-Parametric Mapping toolbox (SnPM13; http://warwick.ac.uk/snpm) to report FWE-corrected $p$ values. Estimations were based on 5000 permutations (Nichols and Holmes, 2002; Smith and Nichols, 2009).

Based on results from previous pain studies, we defined several a priori regions of interest. In the brain the following regions were considered: thalamus, primary somatosensory cortex (SI), secondary somatosensory cortex (SII), insula, ACC, striatum, amygdala, hypothalamus, midbrain, and the cerebellum. For these regions the multiple-comparison correction was restricted to 3D structural masks obtained from the Wake Forest University PickAtlas (WFU_PickAtlas, release 3.0.4). For the spinal cord, correction was limited to a sphere of a $5 \mathrm{~mm}$ radius centered on the peak coordinate from the "main effect of pain" contrast from a previous spinal fMRI study that applied noxious heat stimuli at exactly the 


\section{$\mathbf{A}$}
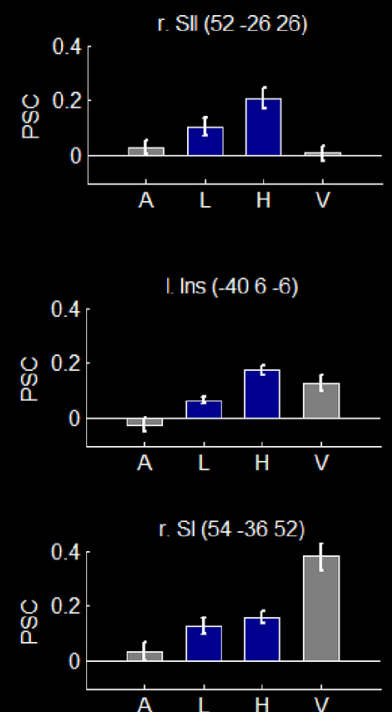

$y=6$

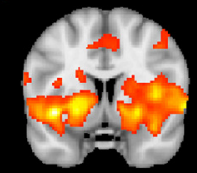

$y=-36$

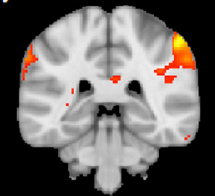

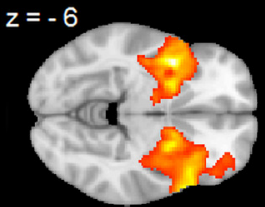

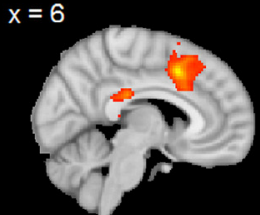

$z=-6$

$z=24$

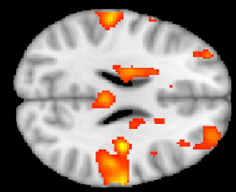

ACC (6 14 44)
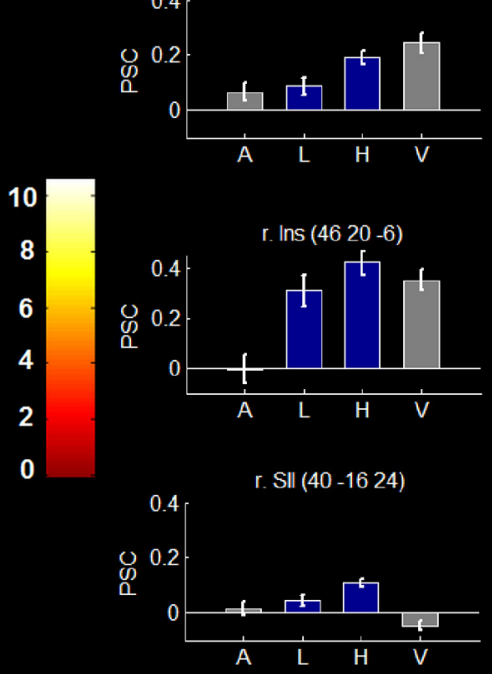

B

Low Intensity
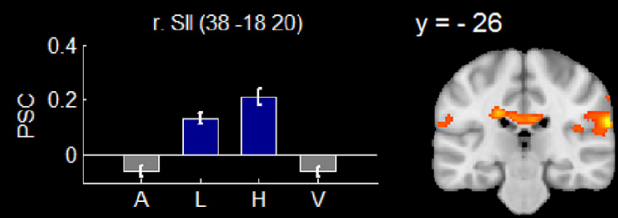

$x=6$
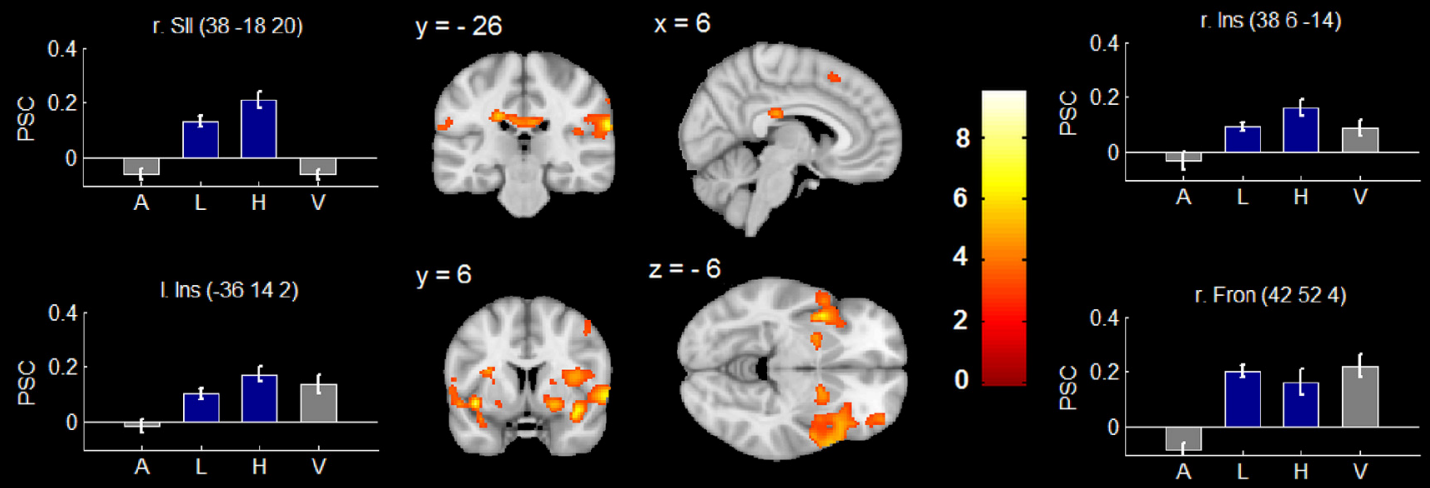

C

\section{High minus Low}
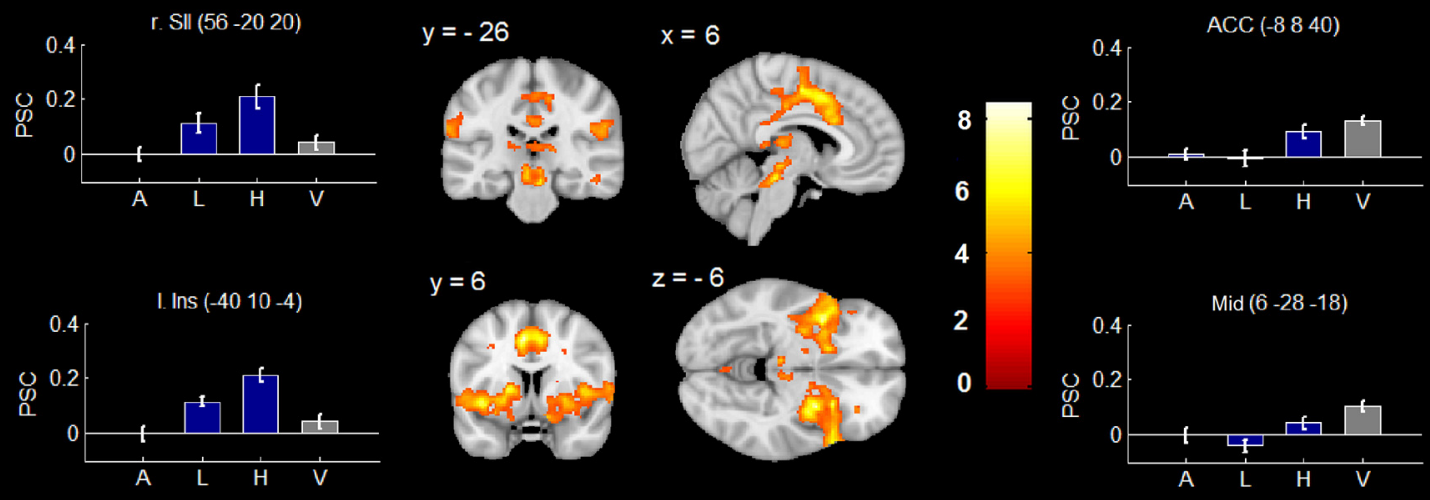

Figure 1. BOLD responses to painful thermal stimulation in the brain. $A, B O L D$ responses corresponding to the high-intensity thermal condition exhibit a typical pattern of pain-sensitive brain regions including thalamus, $\mathrm{SI}, \mathrm{SIl}$, insula, ACC, striatum, and the midbrain. $\boldsymbol{B}$, During the low thermal intensity condition a similar but much weaker pattern exists. $C$, The pain-specific contrast high-intensity stimulation $>$ low-intensity stimulation reveals the same pattern of brain regions constituting the pain network in the brain. Percentage signal changes (PSCs) were extracted from peak voxels of the respective contrast. Error bars indicate SE. For a complete list of brain areas, see Table 1. No significant BOLD responses were observed for the contrast low intensity $>$ high intensity. The color bars indicate $t$ values, and the visualization threshold is set to $p<0.005 . x, y$, and $z$ indicate MNI coordinates. A, anticipation; L, low-intensity thermal stimulation; H, high-intensity thermal stimulation; $\mathrm{V}$, visual analog scale rating; Fron, middle frontal gyrus; Ins, insular cortex; Mid, midbrain. 
same site (Sprenger et al., 2012). Outside a priori regions of interest FWE correction was performed for all voxels within the respective subvolume.

Activations in the brain are reported using $x, y, z$ coordinates in the MNI standard space. Activations in the spinal cord are assigned to the spinal segment, side, and anterior/posterior part of the spinal cord (referred to as ventral/dorsal horn).

For illustration purposes, statistical maps of the brain are thresholded at $p<0.005$ uncorrected for multiple comparisons. To illustrate results from the spinal cord, we also show statistical maps thresholded at $p<$ 0.005 uncorrected for multiple comparisons and additionally statistical maps thresholded at $p<0.05$ uncorrected for multiple comparisons.

\section{Results}

\section{Behavioral results}

All participants exhibited normal heat pain thresholds at the site of stimulus application $\left(45.1 \pm 0.6^{\circ} \mathrm{C}\right.$, mean \pm SEM). Highintensity thermal stimuli were perceived as moderately painful across participants $(56.7 \pm 4.9$, mean \pm SEM), while lowintensity heat stimuli generated only a weak pain perception $(29.8 \pm 4.6)$. We observed significantly higher pain ratings during the high thermal intensity condition compared with the low thermal intensity condition $\left(t_{(16)}=8.40, p<0.05\right)$. Pain ratings remained stable over the experimental time course (highintensity session 1: $55.0 \pm 4.6$; high-intensity session 2: $58.7 \pm$ 4.6; high-intensity session 3: $56.5 \pm 5.0$; low-intensity session 1 : $32.2 \pm 4.5$; low-intensity session $2: 29.1 \pm 5.0$; low-intensity session 3: $28.2 \pm 5.4$ ).

\section{Neuroimaging results}

$B O L D$ responses in the brain

We first tested for BOLD responses in the brain related to highintensity thermal stimulation (high intensity $>$ baseline) and observed a typical pattern of brain regions frequently reported in response to noxious heat stimulation. This includes SI, the bilateral insula, ACC, the bilateral striatum, the bilateral amygdala, and the cerebellum (Fig. $1 A$ ).

In response to low-intensity thermal stimulation (low intensity $>$ baseline) we observed a similar but much weaker pattern of pain-related brain regions including SI, SII, the bilateral insula, and the bilateral striatum (Fig. $1 B$ ).

To reveal neuronal responses that are specifically related to pain perception, we next tested for BOLD responses that are significantly stronger during the high thermal intensity condition compared with the low thermal intensity condition (high intensity $>$ low intensity). This contrast yielded significant activations in the contralateral thalamus, SII, ACC, the bilateral insula, the bilateral striatum, the amygdala, the midbrain, and the cerebellum (Fig. 1C).

We did not observe any BOLD responses to low thermal intensity stimulation that are significantly stronger than the responses to high-intensity stimulation (low intensity $>$ high intensity). We also did not observe a correlation between the neuronal responses to thermal stimulation and the corresponding behavioral pain ratings. A comprehensive list of peak coordinates and statistical values of significant BOLD activations in the brain can be obtained from Table 1 .

\section{$B O L D$ responses in the spinal cord}

In the spinal cord we observed a significant BOLD response related to high-intensity thermal stimulation (high intensity $>$ baseline) in the ipsilateral DH of spinal segment C6 $\left(t_{(16)}=5.15\right.$, pseudo- $t_{(16)}=5.15, p=0.0084$; Figure $\left.2 A, B, E, G\right)$. The peak of the BOLD response to the low-intensity condition (low inten-
Table 1. BOLD responses to painful thermal stimulation in the brain

\begin{tabular}{|c|c|c|c|c|c|c|}
\hline Region & $x$ & $y$ & $Z$ & $p$ value & $t_{(16)}$ & $P$ \\
\hline \multicolumn{7}{|l|}{ High intensity $>$ baseline } \\
\hline \multirow[t]{4}{*}{ SI } & 56 & -40 & 52 & 0.0002 & 6.63 & \\
\hline & 64 & -18 & 22 & 0.0002 & 6.29 & \\
\hline & 58 & -32 & 40 & 0.0108 & 4.27 & \\
\hline & -62 & -24 & 26 & 0.0022 & 6.76 & \\
\hline \multirow[t]{4}{*}{ SII } & 58 & -24 & 20 & 0.0002 & 4.21 & \\
\hline & 48 & -30 & 24 & 0.0002 & 4.76 & \\
\hline & 38 & -22 & 18 & 0.0004 & 4.73 & \\
\hline & -58 & -30 & 20 & 0.0150 & 3.30 & \\
\hline \multirow[t]{6}{*}{ Insula } & 40 & -14 & 12 & 0.0004 & 6.40 & \\
\hline & 42 & 16 & -8 & 0.0004 & 5.26 & \\
\hline & 40 & 0 & 8 & 0.0004 & 5.45 & \\
\hline & -42 & 10 & -4 & 0.0002 & 7.38 & \\
\hline & -32 & 20 & 4 & 0.0010 & 5.67 & \\
\hline & -34 & 10 & -16 & 0.0026 & 4.72 & \\
\hline \multirow[t]{3}{*}{$\mathrm{ACC}$} & 2 & 14 & 38 & 0.0008 & 4.44 & \\
\hline & -2 & 14 & 38 & 0.0028 & 4.37 & \\
\hline & -2 & 22 & 30 & 0.0118 & 3.72 & \\
\hline \multirow[t]{5}{*}{ Striatum } & 26 & 4 & -8 & 0.0002 & 7.25 & \\
\hline & 12 & 0 & 14 & 0.0278 & 3.17 & \\
\hline & -22 & 8 & -2 & 0.0002 & 8.06 & \\
\hline & -16 & -12 & 24 & 0.0086 & 4.25 & \\
\hline & -10 & 8 & 4 & 0.0170 & 4.83 & \\
\hline \multirow[t]{2}{*}{ Amygdala } & 22 & 0 & -14 & 0.0002 & 5.27 & \\
\hline & -24 & 0 & -16 & 0.0078 & 3.82 & \\
\hline Hypothalamus & -8 & -6 & -4 & 0.0206 & 4.45 & \\
\hline \multirow[t]{2}{*}{ Cerebellum } & -26 & -70 & -20 & 0.0010 & 5.11 & \\
\hline & -10 & -82 & -20 & 0.0398 & 3.61 & \\
\hline Superior temporal lobe & 60 & 10 & -2 & 0.0002 & 7.34 & \\
\hline \multicolumn{7}{|l|}{ Low intensity $>$ baseline } \\
\hline \multirow[t]{2}{*}{ SI } & 64 & -20 & 24 & 0.0004 & 5.77 & \\
\hline & 56 & -40 & 52 & 0.0006 & 4.78 & \\
\hline SII & 38 & -22 & 18 & 0.0008 & 4.63 & \\
\hline \multirow[t]{2}{*}{ Insula } & 40 & 20 & -4 & 0.0004 & 4.54 & \\
\hline & -42 & 12 & -6 & 0.0002 & 4.39 & \\
\hline \multirow[t]{3}{*}{ Striatum } & 32 & 10 & 8 & 0.0024 & 4.14 & \\
\hline & 22 & 6 & -10 & 0.0424 & 4.84 & \\
\hline & -22 & 2 & -10 & 0.0130 & 4.34 & \\
\hline Inferior frontal gyrus & 42 & 52 & 4 & 0.0008 & 9.29 & \\
\hline
\end{tabular}

$\begin{array}{lrrrrrr}\text { High intensity }>\text { low intensity } & & & & & & \\ \text { Thalamus } & 4 & -20 & 12 & 0.0006 & 3.84 & 5.75 \\ & 18 & -24 & 16 & 0.0384 & 3.01 & 3.76 \\ & 12 & -16 & -2 & 0.0388 & 4.31 & 3.75 \\ \text { SI } & 54 & -26 & 22 & 0.0050 & 4.36 & 4.94 \\ & -62 & -22 & 24 & 0.0436 & 3.83 & 4.09 \\ \text { SII } & 54 & -26 & 22 & 0.0010 & 4.36 & 4.35 \\ \text { Insula } & 44 & 12 & -6 & 0.0010 & 3.92 & 5.46 \\ & -42 & 10 & -4 & 0.0002 & 7.08 & 6.67 \\ & -36 & 0 & 10 & 0.0374 & 3.48 & 3.56 \\ \text { ACC } & 4 & 4 & 44 & 0.0006 & 5.28 & 5.37 \\ & -2 & 2 & 44 & 0.0002 & 5.10 & 5.95 \\ \text { Midbrain } & 12 & -16 & -2 & 0.0508 & 3.57 & 4.03 \\ \text { Striatum } & 28 & 0 & -8 & 0.0002 & 7.63 & 6.46 \\ & -28 & -2 & 0 & 0.0006 & 7.68 & 5.87 \\ \text { Amygdala } & 24 & 0 & -14 & 0.0052 & 3.40 & 4.38 \\ \text { Cerebellum } & -32 & -56 & -22 & 0.0078 & 4.52 & 4.65\end{array}$

Contrasts are listed according to the appearance in the main text. Coordinates are denoted by $x, y, z$ in millimeters (MNI space). $P$ values are corrected for multiple comparisons correction using the FWE approach. The strength of activation is expressed in $t$ scores and pseudo- $t$ scores, respectively ( $\mathrm{df}=16$ ).

sity $>$ baseline) coincided with the peak of the high-intensity condition (Fig. $2 B, C, G$ ). The response to the low-intensity heat stimulation, however, failed significance $\left(t_{(16)}=2.33\right.$, pseudo$\left.t_{(16)}=1.83, p=0.4942\right)$. 


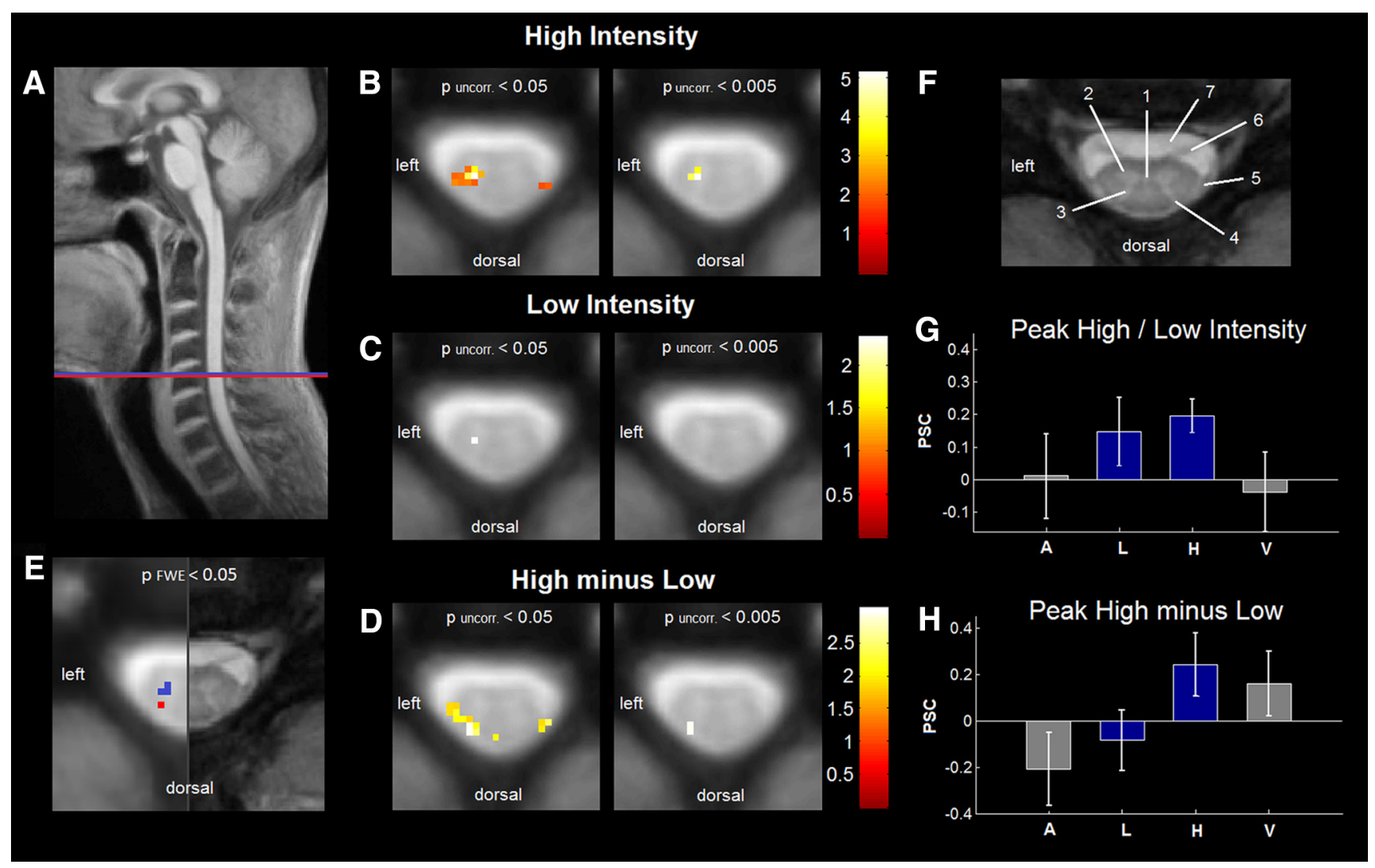

Figure 2. BOLD responses to painful thermal stimulation in the spinal cord. $A$, Brainstem and spinal portions of the average structural image, with the blue line indicating the spinal level of the transverse section in $\boldsymbol{B}$ and $\boldsymbol{C}$, and with the red line (1 $\mathrm{mm}$ below) indicating the spinal level of the transverse section in $\boldsymbol{D}$. The level corresponds to the segment C6. $\boldsymbol{B}, \boldsymbol{C}$, Pain-related BOLD responses during the high-intensity $(\boldsymbol{B})$ and low-intensity $(\boldsymbol{C})$ thermal stimulation thresholded at $p<0.05$ and $p<0.005$ uncorrected for multiple comparisons. Responses are located ipsilateral to the side of stimulation in the deeper sections of the DH of the spinal segment C6. D, BOLD responses that are significantly stronger during the high-intensity thermal stimulation compared with the low thermal intensity stimulation were observed in the superficial layers of the spinal cord ipsilateral to the side of stimulation. $\boldsymbol{E}$, Spatial relationship between significantly activated voxels and substructures of the spinal cord. Clusters in the left part of the image are overlaid on the mean structural MEDIC image; the right part of the image shows an individual normalized MEDIC image to illustrate substructures of the spinal cord. While we observed BOLD responses to the high-intensity thermal stimulation (blue) in central portions of the spinal cord at the transition of the DH to the ventral horn, responses that are significantly stronger during high-intensity stimulation compared with low-intensity stimulation (red) were located in the outer layers of the DH close to the Lissauer tract. The visualization threshold is set to $p<0.05$ corrected for multiple comparisons. For illustration purposes both clusters are projected into one section. Blue refers to the spinal level indicated by the blue line in $A$, and red refers to the spinal level indicated by the red line in $\boldsymbol{A . F}$, Spinal cord anatomy: (1) central canal, (2) ventral horn, (3) dorsal horn, (4) Lissauer tract, (5) dorsal root, (6) ventral root, (7) CSF. G, H, Percentage signal changes (PSCS) were extracted from the peak voxel of the high thermal intensity stimulation contrast $(\boldsymbol{G})$ and the high $>$ low thermal intensity stimulation contrast $(\boldsymbol{H})$. The color bars indicate $t$ values. Error bars indicate SE. A, anticipation; L, low-intensity thermal stimulation; $\mathrm{H}$, high-intensity thermal stimulation; $\mathrm{V}$, visual analog scale rating.

Next, we tested for spinal BOLD responses that are significantly stronger during high-intensity thermal stimulation compared with low-intensity thermal stimulation (high intensity $>$ low intensity). We observed a cluster in the ipsilateral DH of the spinal segment C6 approximately at the same level of the response to the high-intensity thermal stimulation $t_{(16)}=2.94$, pseudo- $t_{(16)}=3.34, p=0.0382$; Fig. $\left.2 A, D, E, H\right)$. While the peak of the high thermal intensity response (high intensity $>$ baseline) was found in the deeper sections of the $\mathrm{DH}$, responses that are significantly stronger during highintensity stimulation compared with low-intensity stimulation were located in the outer layers of the $\mathrm{DH}$ close to the Lissauer tract (Fig. 2E).

We also tested for negative responses to high- and to lowintensity thermal stimulation and for responses that are significantly higher during low-intensity stimulation compared with high-intensity stimulation (low intensity $>$ high intensity), but we did not observe any significant effects. Also in the spinal cord we did not observe a correlation between the BOLD responses to painful stimulation and the behavioral data.
Functional connectivity between the spinal cord and the brain

To assess the functional connectivity between the spinal cord and the brain, we correlated the spinal time series obtained from the respective seed voxel with all voxels in the brain after removing those variance proportions from the brain's time series that can be attributed to the experimental regressors used in our initial GLM analysis.

For the seed voxel determined by the "main effect of pain" in the spinal cord we did not observe any significant correlations between the spinal cord and the brain.

Subsequently, we used the peak of the high-intensity $>$ lowintensity contrast in the spinal cord (see above) as the seed voxel. We observed a significant positive correlation between the spinal seed voxel and the thalamus, SI, the bilateral insula, the bilateral striatum, the bilateral amygdala, the hypothalamus, and the midbrain (in the area of the PAG and bilaterally in the in area of the substantia nigra; Fig. 3A). We did not observe any negative correlations between the spinal time series and the time series in the brain. A list of peak coordinates and corresponding statistical values can be obtained from Table 2. 

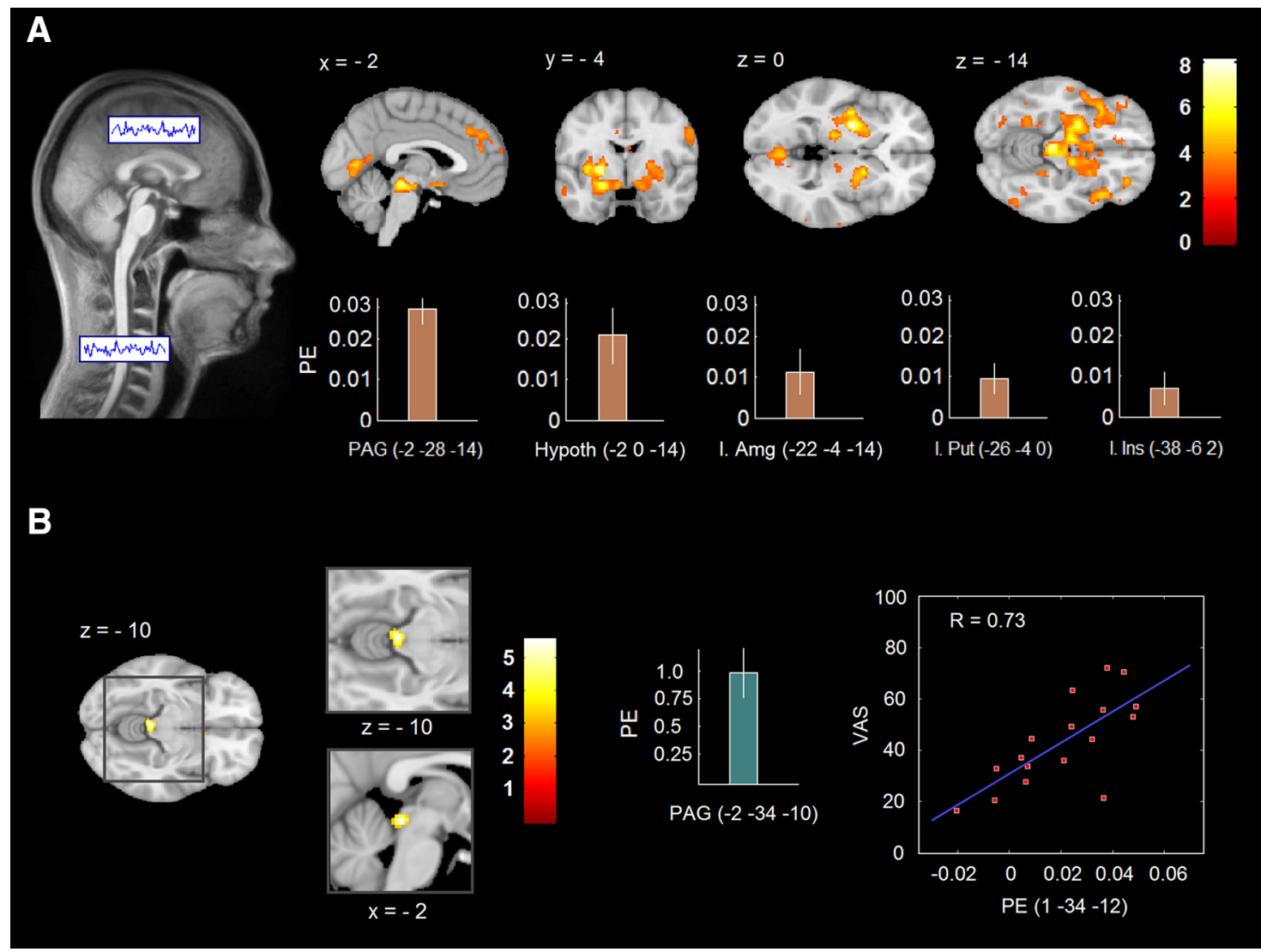

Figure 3. Functional connectivity between the spinal DH and the brain based on adjusted time series correlations. $A$, Based on the seed voxel in the spinal cord determined by the high-intensity $>$ low-intensity contrast, functional coupling is observed between the C6-DH and the SI, SII, the bilateral insula, striatum, PAG/midbrain, bilateral amygdala, and hypothalamus. B, Positive correlation between the individual mean pain rating and the individual strength of functional connectivity in the PAG. The scatter plot is obtained from the peak of the overlap between the area showing a positive correlation of behavioral ratings and connectivity strength and the area showing a significant connectivity with the spinal $\mathrm{DH}$ as such. The visualization threshold is set to $p<0.005$, uncorrected for multiple comparisons. The color bars indicate $t$ values. Error bars indicate SE. PE, parameter estimates; Hypoth, hypothalamus; Amg, amygdala; Put, putamen; Ins, Insula.

Table 2. Brain regions exhibiting functional connectivity with the spinal seed

\begin{tabular}{lrrrlll}
\hline Region & $x$ & $y$ & $z$ & $p$ value & $t_{(16)}$ & Pseudo- $t_{(16)}$ \\
\hline Thalamus & 6 & -22 & -2 & 0.0230 & 2.76 & 4.75 \\
SI & 64 & -20 & 32 & 0.0060 & 4.84 & 4.64 \\
Insula & -38 & -6 & 2 & 0.0254 & 5.75 & 3.92 \\
& -40 & 12 & -12 & 0.0294 & 2.94 & 3.84 \\
Striatum & 26 & 0 & 0 & 0.0066 & 4.41 & 5.32 \\
& -24 & -2 & 0 & 0.0008 & 7.38 & 7.56 \\
& -4 & 6 & -12 & 0.0092 & 3.67 & 5.14 \\
Amygdala & 14 & -6 & -18 & 0.0020 & 3.94 & 4.73 \\
& -22 & -2 & -18 & 0.0012 & 4.94 & 5.00 \\
& -14 & -8 & -16 & 0.0012 & 4.69 & 4.75 \\
Hypothalamus & -2 & 0 & -14 & 0.0058 & 3.58 & 4.08 \\
& -4 & -12 & -12 & 0.0412 & 3.35 & 2.88 \\
Midbrain & 0 & -30 & -14 & 0.0012 & 6.77 & 7.42 \\
& -10 & -10 & -16 & 0.0014 & 5.89 & 6.90 \\
& 16 & -16 & -18 & 0.0094 & 5.11 & 5.57 \\
\hline
\end{tabular}

Results are listed according to the appearance in the main text. Coordinates are denoted by $x, y, z$ in millimeters (MNI space). $P$ values are corrected for multiple comparisons correction using the FWE approach.

Finally, we correlated the individual strength of coupling between the spinal seed voxel and the voxels in the brain subvolume with the individual mean pain ratings. We observed a positive correlation between the individual strength of functional connectivity and the mean behavioral pain ratings in the PAG ([ -2 $-36-10], t_{(16)}=5.73$, pseudo- $t_{(16)}=4.77, p=0.02$; Figure $3 B$ ). No negative correlations between the behavioral ratings and the functional connectivity were observed.

\section{Discussion}

In this study, we investigated functional connectivity between the spinal cord and the brain during pain. Our data show significant interactions between the $\mathrm{DH}$ of the spinal cord at $\mathrm{C} 6$ and several brain structures including the thalamus, primary somatosensory cortex, and bilateral insula. More importantly, we also observed significant functional connectivity with key structures of the descending pain modulatory system such as the PAG, hypothalamus, and the amygdala. The individual strength of the DH-PAG coupling was significantly correlated with the individual pain ratings and thus indicates the functional relevance of this system during physiological pain processing. From a methodological point of view, we demonstrate the feasibility of combined brainspinal $\mathrm{fMRI}$ at $3 \mathrm{~T}$ during using a novel MRI acquisition protocol that was specifically designed to allow two independent fields of view and parameter settings that can be adapted to the particular challenges of the respective region.

While a number of studies reported concurrent brainstem and cortical BOLD changes (Komisaruk et al., 2002; DaSilva et al., 2002; Stankewitz et al., 2010; Mainero et al., 2011), this is to our knowledge the first study investigating spinal and cortical responses to pain during the same experiment. In the brain we observed a typical pattern of BOLD responses to the highintensity thermal stimulation (widespread bilateral insula activation extending to the parietal operculum; ACC, SI, SII, and cerebellar activation), which can be frequently observed in response to acute painful stimulation in healthy volunteers (Peyron 


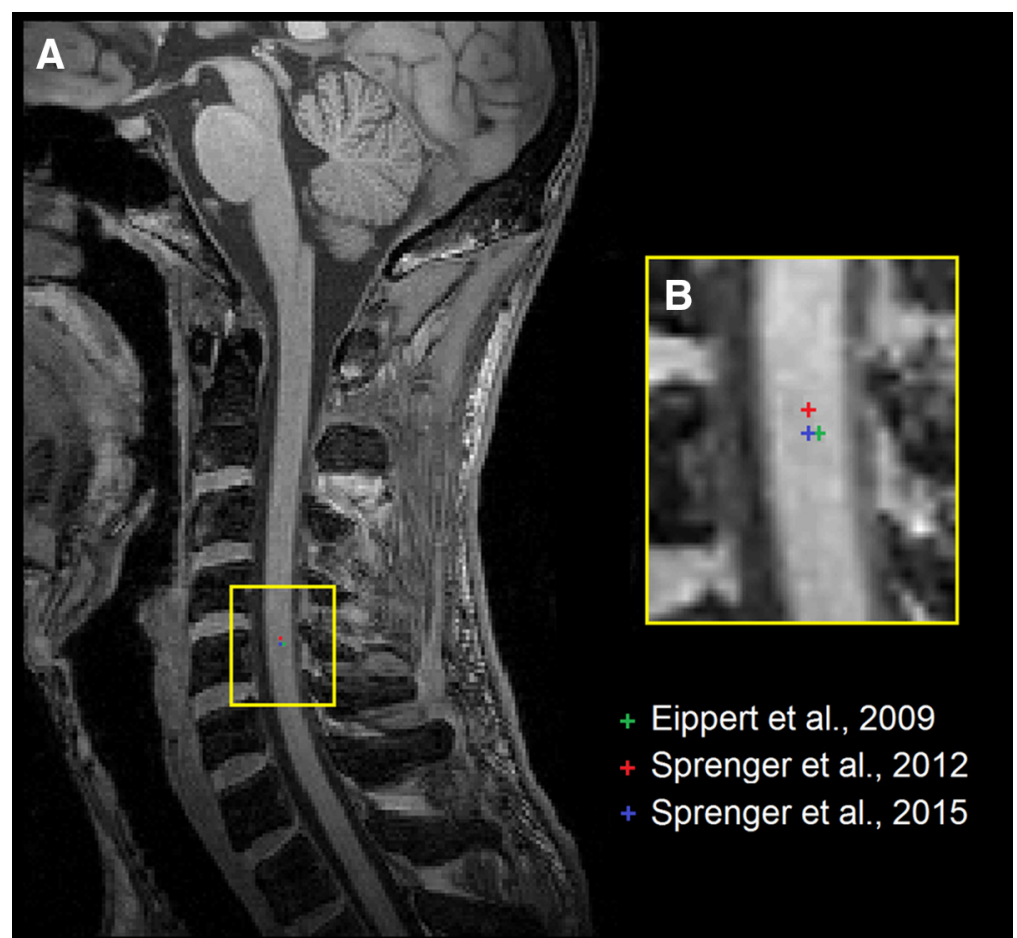

Figure 4. Spinal level of the main effect of pain in three independent fMRI studies that used the same site of stimulation. $\boldsymbol{A}$ Eippert et al., 2009 (green), Sprenger et al., 2012 (red), and the current study (blue) applied painful thermal stimuli with a Peltier thermode to the left radial forearm. The $y$ and $z$ coordinates of the "main effect of pain" activation are shown overlaid on the single subject T1 image that was used for the normalization procedure in these studies. $\boldsymbol{B}$, For illustration purposes the slightly different $x$-coordinates were projected into the median sagittal plane.

et al., 2000; Apkarian et al., 2005; Wager et al., 2013). It has been argued that a similar pattern can also be detected in response to other modalities, representing a common salience detection network (Legrain et al., 2011), but also the contrast high $>$ low intensity revealed a very similar pattern pointing toward a more specific involvement of these regions during pain processing.

As in previous studies (Eippert et al., 2009; Sprenger et al., 2012; Geuter and Büchel, 2013), we stimulated the left radial forearm in an area that is most likely part of the dermatome C6 (Lee et al., 2008) and observed corresponding BOLD responses in the ipsilateral DH of the spinal segment C6. Although in individual cases deviations in the segmental innervation pattern might exist, e.g., due to anastomoses between spinal nerve roots (Moriishi et al., 1989), such an assignment can be made in most cases by determining the position of the spinal rootlets in the individual high-resolution structural images and, moreover, on a group level based on information regarding the anatomical relationship between the spinal segments and the vertebral bodies (Kim et al., 2012; Cadotte et al., 2014).

As the normalization procedure in the current study is based on the same individual spinal single-subject T1-template as in two previous studies stimulating the same body site (Eippert et al., 2009; Sprenger et al., 2012), a comparison of the localization of the peak voxels is permissible (Fig. 4). Additionally, a recent study from Geuter and Büchel (2013) used the same site of stimulation but a slightly different normalization procedure. Reassuringly, all four independent studies found the peak response of the main effect of pain approximately at the same spinal level in the segment C6, ipsilateral to the side of stimulation. By using a very similar methodological approach these studies demonstrate that
BOLD-fMRI of the human spinal cord provides good reproducibility when studying thermal pain.

Although the majority of studies investigating responses to noxious stimulation in the spinal cord found a predominant ipsilateral BOLD response (Eippert et al., 2009; Brooks et al., 2012; Sprenger et al., 2012; Nash et al., 2013), studies frequently also observed contralateral responses (Summers et al., 2010; Brooks et al., 2012; Geuter and Büchel, 2013), which is plausible with regard to propriospinal mechanisms such as interneuron activity and reflex arcs. In the current study we only observed ipsilateral but no contralateral BOLD responses. This is most probably explained by a slightly reduced sensitivity due to the lower temporal SNR of the combined acquisitions (Finsterbusch et al., 2013). In line with this, the strongest response to the low-intensity condition was expectedly found at the same site as the peak of the high-intensity condition but failed significance.

Responses to the high-intensity and to the low-intensity stimulation were observed in deeper sections of the $\mathrm{DH}$ and showed a comparable signal change, while responses that are significantly stronger during the high-intensity condition compared with low intensity were found in the superficial layers of the $\mathrm{DH}$. This might point toward the known distribution of the different types of pain-related secondary neurons within the DH (Willis, 2007; Todd, 2010), but given the limitations of our method regarding in-plane resolution and precision of the normalization procedure, and the fact that the source of the spinal BOLD response is not fully understood yet (Giove et al., 2004; Summers et al., 2014), this observation needs confirmation in future studies.

To reveal functional connectivity between the brain and the spinal cord we correlated adjusted time series obtained from the $\mathrm{DH}$ with the time series in the brain while accounting for taskinduced variance. Although a variety of network-modeling approaches exist, this is based on the finding that correlation-based approaches are quite sensitive in detecting functional networks, even in the presence of a higher amount of noise (Smith et al., 2011), while more complex approaches such as lag-based methods (Granger, 1969) or dynamic causal modeling (Friston et al., 2003) might suffer from the higher level of noise at the spinal level, the potential effects of HRF variability, and the rather long TR. This approach reveals functional connectivity based on residual fluctuations of neuronal activity after removing taskinduced variance (Arfanakis et al., 2000; Fair et al., 2007), therefore, avoiding the detection of connectivity based on common activation-induced effects (Poldrack et al., 2011).

The connectivity analysis revealed functional coupling between the C6-DH and typical ascending thalamocortical pain pathways such as the thalamus, the primary somatosensory cortex, and the bilateral insula. Although the spatial extent of brain areas that showed functional connectivity with the spinal cord was generally smaller compared with areas that showed pain-related activation as such, we observed a clear overlap between both. 
More importantly, we identified functional coupling between the spinal cord and brain regions that are well known to be involved in descending pain modulation such as the PAG, the hypothalamus, and the amygdala (Millan, 2002; Fields, 2004). Although we refrain from estimating directionality of the interaction (which is difficult based on fMRI measures), our analysis elucidates the functional relationship between these pain modulatory regions and the spinal cord.

We did not observe any significant correlations if we used the spinal "main effect of pain" peak as the seed voxel. This might point toward the different functional roles of superficial and deeper spinal laminae (Le Bars, 2002; Todd, 2010). However, findings regarding the substructures of the $\mathrm{DH}$ should be interpreted with caution.

Interestingly, we observed a positive correlation between the fMRI signal in the DH and the PAG. Although activity of ON and OFF cells in the PAG-rostral ventromedial medulla axis might complicate the interpretation of signal changes within this system, most fMRI studies interpreted increased activity in the PAG as a top-down inhibitory action (Wiech et al., 2008). Tracey et al. (2002), for example, observed an increased PAG activity during hypoalgesia through distraction, while we observed a corresponding inhibition of BOLD responses on the spinal level (Sprenger et al., 2012). Together with the fact that we did not observe an activation of the PAG compared with baseline, it can be assumed that the PAG did not cause a strong inhibitory effect on the $\mathrm{DH}$ in the current paradigm. Furthermore, comparable thermal stimuli are neither behaviorally modulated by the opioid antagonist naloxone nor is PAG activity altered in this context (Schoell et al., 2010). However, this does not exclude that the PAG exerted an inhibitory action on the DH. The findings might be best explained by a negative feedback loop between the $\mathrm{DH}$ and the PAG in the absence of a strong top-down inhibition, where the PAG counteracts fluctuations of the nociceptive signal ascending from the spinal cord.

Importantly, our analysis revealed a positive correlation of the individual strength of connectivity within this descending pain modulatory pathway/feedback loop with the behavioral pain ratings (and not within an ascending pain pathway), pointing to the functional relevance of this system during the processing of physiological nociceptor pain.

While previous studies indicated a functional role of the PAG in different models of endogenous analgesia, our data show that the DH-PAG coupling already plays a functional role during physiological pain signaling. This is in line with the notion that the PAG-DH system is not only selectively activated during conditions that require pain inhibition but rather continuously balancing the organism between pain facilitation and pain inhibition (Millan, 2002; Fields, 2004; Heinricher et al., 2009). In the future, it will be interesting to study the PAG-DH connectivity in experimental models of endogenous analgesia and clinical conditions that have been attributed to an attenuated descending pain inhibition with combined brain-spinal fMRI.

\section{References}

Apkarian AV, Bushnell MC, Treede RD, Zubieta JK (2005) Human brain mechanisms of pain perception and regulation in health and disease. Eur J Pain 9:463-484. CrossRef Medline

Arfanakis K, Cordes D, Haughton VM, Moritz CH, Quigley MA, Meyerand ME (2000) Combining independent component analysis and correlation analysis to probe interregional connectivity in fMRI task activation datasets. Magn Reson Imaging 18:921-930. CrossRef Medline

Ashburner J, Friston KJ (2005) Unified segmentation. Neuroimage 26:839851. CrossRef Medline
Barry RL, Smith SA, Dula AN, Gore JC (2014) Resting state functional connectivity in the human spinal cord. Elife 3:e02812. CrossRef Medline

Birn RM (2012) The role of physiological noise in resting-state functional connectivity. Neuroimage 62:864-870. CrossRef Medline

Blamire AM, Rothman DL, Nixon T (1996) Dynamic shim updating: a new approach towards optimized whole brain shimming. Magn Reson Med 36:159-165. CrossRef Medline

Brooks JC, Beckmann CF, Miller KL, Wise RG, Porro CA, Tracey I, Jenkinson M (2008) Physiological noise modelling for spinal functional magnetic resonance imaging studies. Neuroimage 39:680-692. CrossRef Medline

Brooks JC, Kong Y, Lee MC, Warnaby CE, Wanigasekera V, Jenkinson M, Tracey I (2012) Stimulus site and modality dependence of functional activity within the human spinal cord. J Neurosci 32:6231-6239. CrossRef Medline

Brooks JCW (2014) Physiological noise modeling and analysis for spinal cord fMRI. In: Quantitative MRI of the spinal cord, Ed 1, pp 240-257. Amsterdam: Elsevier.

Büchel C, Geuter S, Sprenger C, Eippert F (2014) Placebo analgesia: a predictive coding perspective. Neuron 81:1223-1239. CrossRef Medline

Cadotte DW, Cadotte A, Cohen-Adad J, Fleet D, Livne M, Mikulis D, Fehlings MG (2014) Resolving the anatomic variability of the human cervical spinal cord: a solution to facilitate advanced neural imaging. International Society for Magnetic Resonance in Medicine, Milan, Italy.

Collignon A, Maes F, Delaere D, Vandermeulen D, Suetens P, Marchal G (1995) Automated multi-modality image registration based on information theory. In: Proceedings of the XIVth International Conference on Information Processing in Medical Imaging. Computation imaging vision, Vol 3, pp. 263-274. Boston: Kluwer Academic.

Cooke FJ, Blamire AM, Manners DN, Styles P, Rajagopalan B (2004) Quantitative proton magnetic resonance spectroscopy of the cervical spinal cord. Magn Reson Med 51:1122-1128. CrossRef Medline

DaSilva AF, Becerra L, Makris N, Strassman AM, Gonzalez RG, Geatrakis N, Borsook D (2002) Somatotopic activation in the human trigeminal pain pathway. J Neurosci 22:8183-8192. Medline

Deckers RH, van Gelderen P, Ries M, Barret O, Duyn JH, Ikonomidou VN, Fukunaga M, Glover GH, de Zwart JA (2006) An adaptive filter for suppression of cardiac and respiratory noise in MRI time series data. Neuroimage 33:1072-1081. CrossRef Medline

Eippert F, Finsterbusch J, Bingel U, Büchel C (2009) Direct evidence for spinal cord involvement in placebo analgesia. Science 326:404. CrossRef Medline

Fair DA, Schlaggar BL, Cohen AL, Miezin FM, Dosenbach NU, Wenger KK, Fox MD, Snyder AZ, Raichle ME, Petersen SE (2007) A method for using blocked and event-related fMRI data to study "resting state" functional connectivity. Neuroimage 35:396-405. CrossRef Medline

Fields H (2004) State-dependent opioid control of pain. Nat Rev Neurosci 5:565-575. CrossRef Medline

Finsterbusch J, Eippert F, Büchel C (2012) Single, slice-specific z-shim gradient pulses improve $\mathrm{T} 2{ }^{\star}$-weighted imaging of the spinal cord. Neuroimage 59:2307-2315. CrossRef Medline

Finsterbusch J, Sprenger C, Büchel C (2013) Combined T2*-weighted measurements of the human brain and cervical spinal cord with a dynamic shim update. Neuroimage 79:153-161. CrossRef Medline

Friston KJ, Buechel C, Fink GR, Morris J, Rolls E, Dolan RJ (1997) Psychophysiological and modulatory interactions in neuroimaging. Neuroimage 6:218-229. CrossRef Medline

Friston KJ, Harrison L, Penny W (2003) Dynamic causal modelling. Neuroimage 19:1273-1302. CrossRef Medline

Geuter S, Büchel C (2013) Facilitation of pain in the human spinal cord by nocebo treatment. J Neurosci 33:13784-13790. CrossRef Medline

Giove F, Garreffa G, Giulietti G, Mangia S, Colonnese C, Maraviglia B (2004) Issues about the fMRI of the human spinal cord. Magn Reson Imaging 22:1505-1516. CrossRef Medline

Granger CW (1969) Investigating causal relations by econometric models and cross-spectral methods. Econometrica 37:424-438. CrossRef

Heinricher MM, Tavares I, Leith JL, Lumb BM (2009) Descending control of nociception: specificity, recruitment and plasticity. Brain Res Rev 60: 214-225. CrossRef Medline

Kim JH, Lee CW, Chun KS, Shin WH, Bae HG, Chang JC (2012) Morphometric relationship between the cervicothoracic cord segments and vertebral bodies. J Korean Neurosurg Soc 52:384-390. CrossRef Medline

Komisaruk BR, Mosier KM, Liu WC, Criminale C, Zaborszky L, Whipple B, 
Kalnin A (2002) Functional localization of brainstem and cervical spinal cord nuclei in humans with fMRI. AJNR Am J Neuroradiol 23:609-617. Medline

Kong Y, Jenkinson M, Andersson J, Tracey I, Brooks JC (2012) Assessment of physiological noise modelling methods for functional imaging of the spinal cord. Neuroimage 60:1538-1549. CrossRef Medline

Le Bars D (2002) The whole body receptive field of dorsal horn multireceptive neurones. Brain Res Brain Res Rev 40:29-44. CrossRef Medline

Lee MW, McPhee RW, Stringer MD (2008) An evidence-based approach to human dermatomes. Clin Anat 21:363-373. CrossRef Medline

Legrain V, Iannetti GD, Plaghki L, Mouraux A (2011) The pain matrix reloaded: a salience detection system for the body. Prog Neurobiol 93:111124. CrossRef Medline

Maieron M, Iannetti GD, Bodurka J, Tracey I, Bandettini PA, Porro CA (2007) Functional responses in the human spinal cord during willed motor actions: evidence for side-and rate-dependent activity. J Neurosci 27: 4182-4190. CrossRef Medline

Mainero C, Zhang WT, Kumar A, Rosen BR, Sorensen AG (2007) Mapping the spinal and supraspinal pathways of dynamic mechanical allodynia in the human trigeminal system using cardiac-gated fMRI. Neuroimage 35: 1201-1210. CrossRef Medline

Mainero C, Boshyan J, Hadjikhani N (2011) Altered functional magnetic resonance imaging resting-state connectivity in periaqueductal gray networks in migraine. Ann Neurol 70:838-845. CrossRef Medline

Millan MJ (2002) Descending control of pain. Prog Neurobiol 66:355-474. CrossRef Medline

Moriishi J, Otani K, Tanaka K, Inoue S (1989) The intersegmental anastomoses between spinal nerve roots. Anat Rec 224:110-116. CrossRef Medline

Morrell G, Spielman D (1997) Dynamic shimming for multi-slice magnetic resonance imaging. Magn Reson Med 38:477-483. CrossRef Medline

Nash P, Wiley K, Brown J, Shinaman R, Ludlow D, Sawyer AM, Glover G, Mackey S (2013) Functional magnetic resonance imaging identifies somatotopic organization of nociception in the human spinal cord. Pain 154:776-781. CrossRef Medline

Nichols TE, Holmes AP (2002) Nonparametric permutation tests for functional neuroimaging: a primer with examples. Hum Brain Mapp 15:1-25. CrossRef Medline

Peyron R, Laurent B, García-Larrea L (2000) Functional imaging of brain responses to pain. A review and meta-analysis (2000). Neurophysiol Clin 30:263-288. CrossRef Medline

Piché M, Cohen-Adad J, Nejad MK, Perlbarg V, Xie G, Beaudoin G, Benali H, Rainville P (2009) Characterization of cardiac-related noise in fMRI of the cervical spinal cord. Magn Reson Imaging 27:300-310. CrossRef Medline

Poldrack RA, Mumford JA, Nichols TE (2011) Handbook of functional MRI data analysis. New York: Cambridge UP.

Porreca F, Ossipov MH, Gebhart GF (2002) Chronic pain and medullary descending facilitation. Trends Neurosci 25:319-325. CrossRef Medline

Reynolds DV (1969) Surgery in the rat during electrical analgesia induced by focal brain stimulation. Science 164:444-445. CrossRef Medline

Rolke R, Baron R, Maier C, Tölle TR, Treede RD, Beyer A, Binder A, Birbaumer N, Birklein F, Bötefür IC, Braune S, Flor H, Huge V, Klug R, Landwehrmeyer GB, Magerl W, Maihöfner C, Rolko C, Schaub C, Scherens A, et al. (2006) Quantitative sensory testing in the German Research Network on Neuropathic Pain (DFNS): standardized protocol and reference values. Pain 123:231-243. CrossRef Medline

Schoell ED, Bingel U, Eippert F, Yacubian J, Christiansen K, Andresen H, May A, Buechel C (2010) The effect of opioid receptor blockade on the neural processing of thermal stimuli. PLoS One 5:e12344. CrossRef Medline

Schroth G, Klose U (1992a) Cerebrospinal fluid flow. I. Physiology of cardiac-related pulsation. Neuroradiology 35:1-9. CrossRef Medline
Schroth G, Klose U (1992b) Cerebrospinal fluid flow. II. Physiology of respiration-related pulsations. Neuroradiology 35:10-15. CrossRef Medline

Smith SM, Nichols TE (2009) Threshold-free cluster enhancement: addressing problems of smoothing, threshold dependence and localisation in cluster inference. Neuroimage 44:83-98. CrossRef Medline

Smith SM, Miller KL, Salimi-Khorshidi G, Webster M, Beckmann CF, Nichols TE, Ramsey JD, Woolrich MW (2011) Network modelling methods for fMRI. Neuroimage 54:875-891. CrossRef Medline

Sprenger C, Bingel U, Büchel C (2011) Treating pain with pain: supraspinal mechanisms of endogenous analgesia elicited by heterotopic noxious conditioning stimulation. Pain 152:428-439. CrossRef Medline

Sprenger C, Eippert F, Finsterbusch J, Bingel U, Rose M, Büchel C (2012) Attention modulates spinal cord responses to pain. Curr Biol 22:1019_ 1022. CrossRef Medline

Stankewitz A, Voit HL, Bingel U, Peschke C, May A (2010) A new trigemino-nociceptive stimulation model for event-related fMRI. Cephalalgia 30:475-485. CrossRef Medline

Stroman PW (2005) Magnetic resonance imaging of neuronal function in the spinal cord: spinal FMRI. Clin Med Res 3:146-156. CrossRef Medline

Stroman PW, Wheeler-Kingshott C, Bacon M, Schwab JM, Bosma R, Brooks J, Cadotte D, Carlstedt T, Ciccarelli O, Cohen-Adad J, Curt A, Evangelou N, Fehlings MG, Filippi M, Kelley BJ, Kollias S, Mackay A, Porro CA, Smith S, Strittmatter SM, et al. (2014) The current state-of-the-art of spinal cord imaging: methods. Neuroimage 84:1070-1081. CrossRef Medline

Summers PE, Ferraro D, Duzzi D, Lui F, Iannetti GD, Porro CA (2010) A quantitative comparison of BOLD fMRI responses to noxious and innocuous stimuli in the human spinal cord. Neuroimage 50:1408-1415. CrossRef Medline

Summers PE, Brooks JCW, Cohen-Adad J (2014) Spinal cord fMRI. In: Quantitative MRI of the spinal cord (Cohen-Adad J, Wheeler-Kingshott C, eds), pp 221-239. San Diego, CA: Academic.

Todd AJ (2010) Neuronal circuitry for pain processing in the dorsal horn. Nat Rev Neurosci 11:823-836. CrossRef Medline

Tracey I, Ploghaus A, Gati JS, Clare S, Smith S, Menon RS, Matthews PM (2002) Imaging attentional modulation of pain in the periaqueductal gray in humans. J Neurosci 22:2748-2752. Medline

Verma T, Cohen-Adad J (2014) Effect of respiration on the B0 field in the human spinal cord at 3T. Magn Reson Med 72:1629-1636. CrossRef Medline

Villanueva L, Le Bars D (1995) The activation of bulbo-spinal controls by peripheral nociceptive inputs: diffuse noxious inhibitory controls. Biol Res 28:113-125. Medline

Wager TD, Atlas LY, Lindquist MA, Roy M, Woo CW, Kross E (2013) An fMRI-based neurologic signature of physical pain. N Engl J Med 368: 1388-1397. CrossRef Medline

Wheeler-Kingshott CA, Stroman PW, Schwab JM, Bacon M, Bosma R, Brooks J, Cadotte DW, Carlstedt T, Ciccarelli O, Cohen-Adad J, Curt A, Evangelou N, Fehlings MG, Filippi M, Kelley BJ, Kollias S, Mackay A, Porro CA, Smith S, Strittmatter SM, et al. (2014) The current state-ofthe-art of spinal cord imaging: applications. Neuroimage 84:1082-1093. CrossRef Medline

Wiech K, Ploner M, Tracey I (2008) Neurocognitive aspects of pain perception. Trends Cogn Sci 12:306-313. CrossRef Medline

Willis WD Jr (2007) The somatosensory system, with emphasis on structures important for pain. Brain Res Rev 55:297-313. CrossRef Medline

Worsley KJ, Evans AC, Marrett S, Neelin P (1992) A three-dimensional statistical analysis for $\mathrm{CBF}$ activation studies in human brain. J Cereb Blood Flow Metab 12:900-918. CrossRef Medline 